\title{
Measuring the properties of extragalactic dust and implications for the Hubble diagram
}

\author{
A. Goobar, L. Bergström, and E. Mörtsell \\ Department of Physics, Stockholm University, SCFAB, 10691 Stockholm, Sweden \\ Received 10 August 2001 / Accepted 2 January 2002
}

\begin{abstract}
Scattering and absorption of light by a homogeneous distribution of intergalactic large dust grains has been proposed as an alternative, non-cosmological explanation for the faintness of type Ia supernovae at $z \sim 0.5$. We investigate the differential extinction for high-redshift sources caused by extragalactic dust along the line of sight. Future observations of type Ia supernovae up to $z \sim 2$, e.g. by the proposed SNAP satellite, will allow the measurement of the properties of dust over cosmological distances. We show that $1 \%$ relative spectrophotometric accuracy (or broadband photometry) in the wavelength interval 0.7-1.5 $\mu \mathrm{m}$ is required to measure the extinction caused by "grey" dust down to $\delta m=0.02 \mathrm{mag}$. We also argue that the presence of grey dust is not necessarily inconsistent with the recent measurement of the brightness of a supernova at $z=1.7$ (SN 1997ff), in the absence of accurate spectrophotometric information of the supernova.
\end{abstract}

Key words. cosmological parameters; dust, extinction; supernovae: general

\section{Introduction}

There is observational evidence (Perlmutter et al. 1999; Riess et al. 1998) that type Ia supernovae, when used as calibrated standard candles, are dimmer at high redshift than can be explained in models without a cosmological constant. At least there seems to be a need for a nonclustered "dark energy" component with a negative coefficient in the equation of state, such as obtained in models with an evolving scalar field ("quintessence") (Steinhardt 2000). Since there are potential systematic effects affecting this interpretation, it is important to investigate alternative explanations. In this note, we investigate dimming due to absorption and scattering on intergalactic dust, as has been proposed by Aguirre (1999a, 1999b) to be a viable explanation for the supernova results. The recent measurements of the CMB small angle anisotropies showing that the universe is most likely flat (de Bernardis et al. 2000; Balbi et al. 2000; Pryke et al. 2001), combined with measurements of $\Omega_{\mathrm{M}} \sim 0.3$ from large scale structure (Peacock et al. 2001) and galaxy cluster evolution (Bahcall \& Fan 1998) makes Aguirre's idea for the origin of the type Ia supernovae faintness at $z \sim 0.5$ unlikely.

Moreover, Aguirre \& Haiman (2000) have shown that the amount of dust required to make the supernova results compatible with a flat universe, as indicated by the CMB results, but without a cosmological constant, is already disfavored by the far-infrared background measured

Send offprint requests to: A. Goobar, e-mail: ariel@physto.se by the DIRBE/FIRAS instruments. However, a sizable grey dust column density capable of biasing the results cannot be excluded with the present knowledge. Precision measurements of "Dark Energy" and "Dark Matter" with high- $z$ supernovae have been proposed see e.g. (Goliath et al. 2001; Mörtsell et al. 2001). The assumption in those studies is that the systematic uncertainties do not exceed $\delta m=0.02$ mag. Thus, in this work we concentrate on the needed relative spectrophotometric accuracy to meet this requirement.

Extinction must be considered for at least four different dust environments:

1. A homogeneous intergalactic dust component. Particular attention must be paid to the possibility that the mechanisms expelling dust from galaxies and clusters destroy the smallest grains, thereby causing very little reddening ("grey dust");

2. A host galaxy dust component;

3. Dust in galaxies between the source and the observer;

4. Milky-Way dust.

Properties such as the extinction scale-length and the wavelength dependence can be different for each case.

The main emphasis of this work is on the calculation of the possible effects from intergalactic dust (1). To avoid observational constraints on reddening, such a component must mainly consist of large dust grains as described in (Aguirre 1999a, 1999b). However, even large-grain dust will cause some reddening, and going to higher redshift 
should enable to distinguish between extinction or a cosmological origin for the faintness of type Ia supernovae at $z \sim 0.5$. The future data sample we have in mind here is the one expected from the SNAP satellite (Perlmutter et al. 2000), which will provide several thousand type Ia supernovae out to $z \sim 1.7$.

In this note we also discuss the light extinction by "normal" dust in either the SN host galaxy (2) or galaxies along the line of sight (3). As we will see, the latter only affects sizably about $1 \%$ of the supernovae at $z \sim 1$.

\section{Spectrophotometric calibrators}

Type Ia supernovae form a remarkably homogeneous class of astronomical objects and are therefore well suited for studying the dust properties over cosmological distances. Figure 1 shows the average brightness at maximum light in $V, R, I$ and $J$-band for type Ia supernovae along with the filter transmission functions used ${ }^{1}$. The magnitudes were calculated for a "standard", $\Lambda$-dominated universe, $\left(\Omega_{\mathrm{M}}, \Omega_{\Lambda}\right)=(0.3,0.7)$, with no dust extinction included. The flux in different band-passes is dust model and cosmology dependent as the total extinction at any wavelength depends on the total dust column depth. This, in turn, depends on cosmological parameters and the assumed density of dust to explain the faintness of supernovae at $z \sim 0.5$. To date, one supernova at $z=0.46$ for which there exists NIR data has been used to place limits on grey dust (Riess et al. 2000).

Other potential sources for spectrophotometric studies of dust are quasars and core collapse supernovae. The SDSS and 2DF collaborations are preparing templates that will eventually include about $10^{5}$ QSOs up to redshift $z \sim 5$. A recent study performed by the SDSS group of a sample of 2200 QSO spectra in the redshift range $0.044<$ $z<4.789$ showed a spectrum-to-spectrum $1 \sigma$ difference of approximately $20 \%$ in the rest system wavelength range 0.1 to $0.85 \mu \mathrm{m}$ (Vanden Berk et al. 2001). By averaging over some 400 QSOs in redshift bins of $\Delta z=0.1$, one could thus possibly achieve a $1 \%$ spectrophotometric measurement, if the scatter is of stochastic nature, i.e. not related to evolutionary effects. Type II supernovae exhibit an approximate black body spectrum and are used for distance estimations through the "Expanding Photosphere Method". Although fainter than type Ia and thus harder to observe at high- $z$, they could potentially be used for probing dust, especially if observed with NGST.

\section{Large "grey" dust grains}

Following the idea of Aguirre (1999a, 1999b), we assume that the intergalactic dust population can be described by a Draine \& Lee model (Draine \& Lee 1984) where the smaller grains have been destroyed by some unspecified process, plausibly connected to the expulsion of the dust from the star-forming galaxies where the dust originated.

\footnotetext{
${ }^{1}$ Spectral template kindly provided by P. Nugent (Nugent et al. 2002).
}
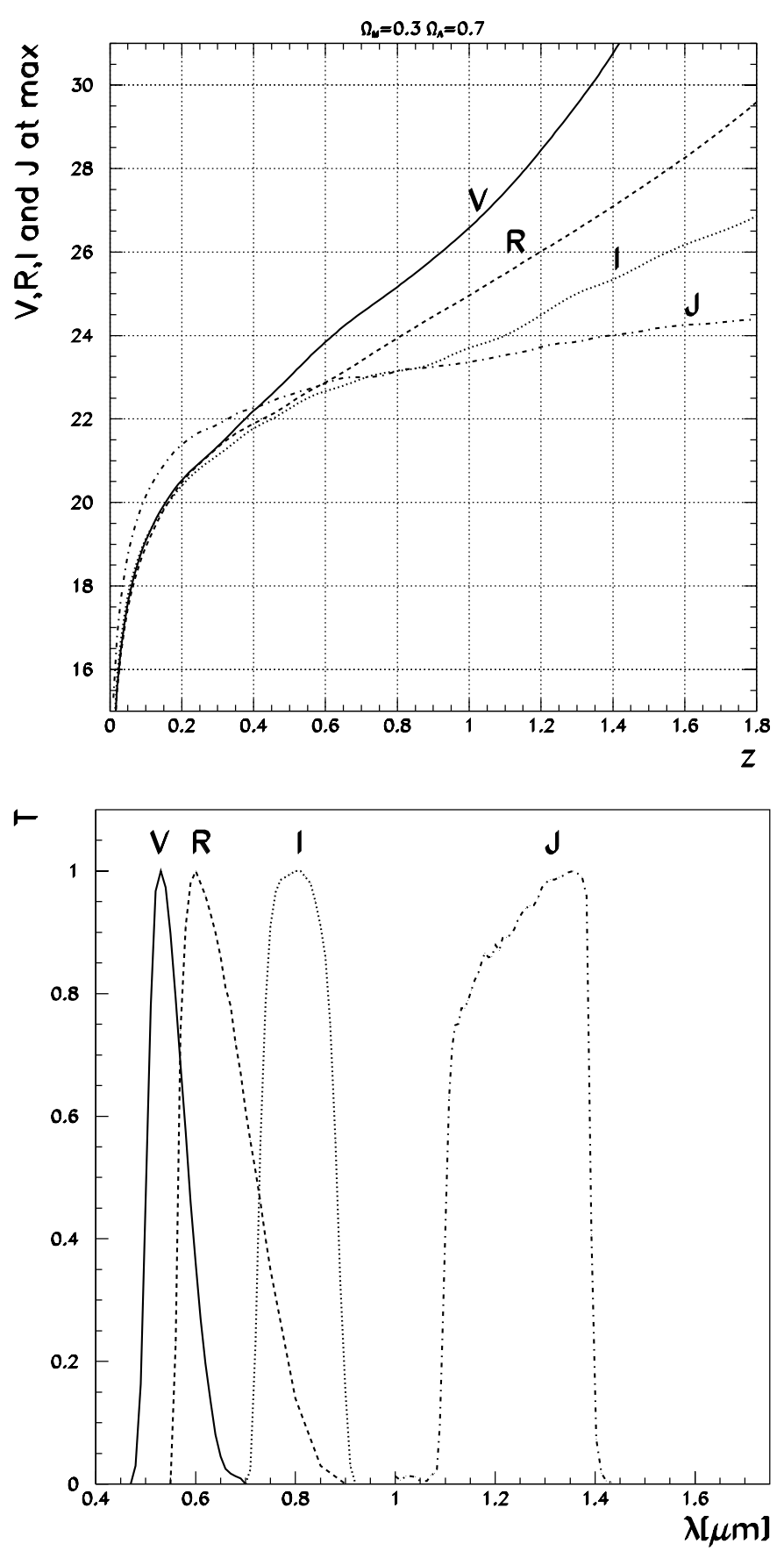

Fig. 1. Top: mean VRIJ magnitudes for type Ia supernovae vs. redshift for $\left(\Omega_{\mathrm{M}}, \Omega_{\Lambda}\right)=(0.7,0.3)$. Bottom: filter transmission functions used.

The reddening parameter $R_{V}$ is defined by

$A_{V}=R_{V} E(B-V)$,

where $A_{V}$ is the wavelength-dependent extinction and

$E(B-V)=(B-V)-(B-V)_{\mathrm{i}}$

with $(B-V)_{\mathrm{i}}$ being the intrinsic (unobscured) color.

The presence of small dust grains enhances the wavelength dependence of extinction. In the adopted parameterization this corresponds to lower values of $R_{V}$. 
Measurements of the restframe $E(B-V)$ for $z \sim 0.5 \mathrm{su}-$ pernovae have been used to set a lower limit $R_{V} \gtrsim 6$ (Perlmutter et al. 1999), see also caveats with regards to this limit in (Aguirre 1999b).

A population of large grains may have an $R_{V}$ parameter as large as 5 to 10 , thus giving a rather achromatic ("grey") absorption.

Typically, the most important types of intergalactic grains are silicates and graphite. The optical properties, of which $R_{V}$ is the most important for our applications, depend to some extent on the actual value chosen for the small-size cutoff $a_{\text {min }}$ in the Draine-Lee power-law size distribution. To make contact with Aguirre's calculations, we will use $a_{\min }$ between 0.08 and $0.12 \mu \mathrm{m}$, corresponding roughly to $R_{V}$ between 5.5 and 9.5 . In the numerical calculations, we use the convenient parameterization of the extinction versus wavelength given by Cardelli et al. (1989). The existence of small dust grains would make the differential extinction effects larger than the ones described here, i.e. easier to identify observationally.

\subsection{Extinction by dust at cosmological distances}

For a given emission redshift $z_{\mathrm{e}}$, the attenuation $\Delta m_{\mathrm{d}}$ at observed wavelength $\lambda_{\mathrm{o}}$ due to dust can be written

$$
\begin{aligned}
& \Delta m_{\mathrm{d}}\left(z_{\mathrm{e}}, \lambda_{\mathrm{o}}\right)= \\
& -2.5 \log _{10}\left[\mathrm{e}^{-C \int_{0}^{z_{\mathrm{e}}} \rho_{\text {dust }}(z) a\left(\lambda_{\circ} /(1+z), R_{V}\right) h(z) \mathrm{d} z}\right],
\end{aligned}
$$

where $\rho_{\text {dust }}(z)$ is the physical dust density at redshift $z$, $a\left(\lambda, R_{V}\right)$ is the wavelength-dependent attenuation ("reddening") parametrized as in (Cardelli et al. 1989), and the cosmology-dependent function $h(z)$ is given by

$h(z)=\frac{1}{H_{0}(1+z) \sqrt{(1+z)^{2}\left(1+\Omega_{\mathrm{M}} z\right)-z(2+z) \Omega_{\Lambda}}}$.

The normalization constant $C$ is related to the overall magnitude of the extinction, which we fix by demanding that a given cosmology reproduces the observed luminosity distance at $z \sim 0.5$, i.e., that dust extinction can explain the dimming of the presently observed supernova sample, otherwise attributed to the "concordance" cosmology $\Omega_{\mathrm{M}}=0.3, \Omega_{\Lambda}=0.7$.

The Monte-Carlo simulation program SNOC (Goobar et al. 2002) was used to perform the integral in Eq. (1) numerically by following individual light paths through a large number of cells containing galaxies and intergalactic dust. Through each cell the background cosmology, the wavelength of the photon and the dust density were updated, and the contributions from each cell added. Note that the model is approximately valid also for a patchy dust distribution, as long as the scale of inhomogeneities is small enough, i.e., $1 / \sqrt{N} \ll 1$ where $N$ is the number of dust clouds intersected by the light-ray.

First we summarize the situation concerning the dependence of the observed magnitudes on the cosmological background model, in the absence of dust. Figure 2

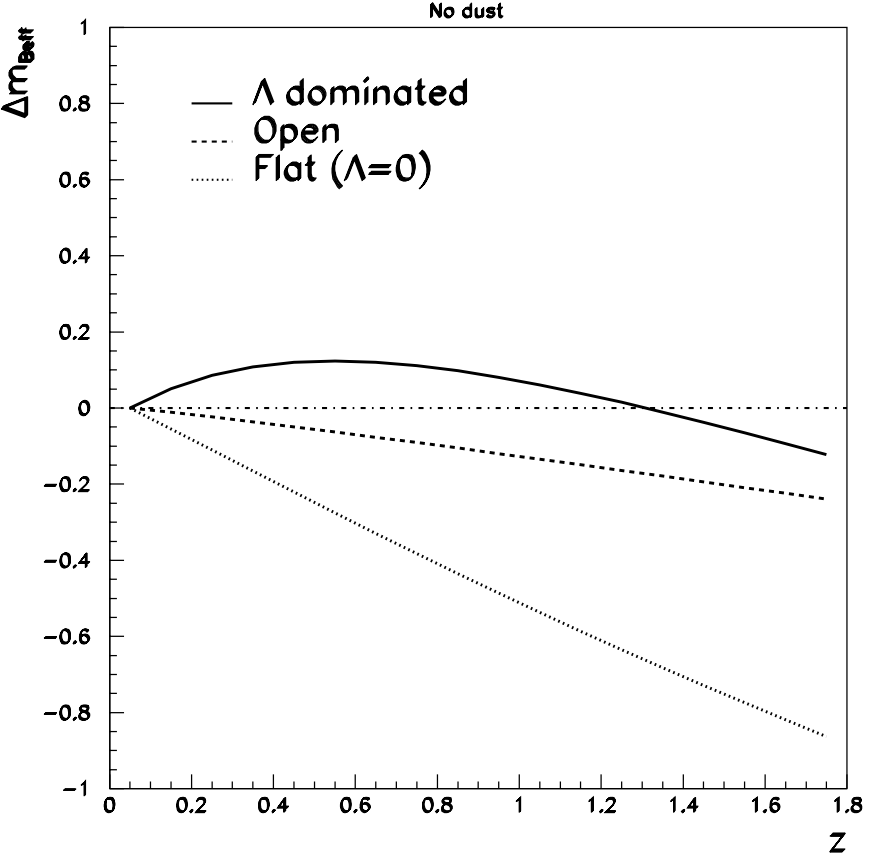

Fig. 2. Differential magnitude for three cosmologies, $\Omega_{\mathrm{M}}, \Omega_{\Lambda}=$ $(0.3,0.7)$ (solid line), $(0.2,0)$ (dashed line) and $(1,0)$ (dotted line), compared with an empty universe, $\left(\Omega_{\mathrm{M}}, \Omega_{\Lambda}\right)=(0,0)$ (horizontal, dash-dotted line).

shows the magnitude difference for three cosmologies, $\left(\Omega_{\mathrm{M}}, \Omega_{\Lambda}\right)=(0.3,0.7),(0.2,0)$ and $(1,0)$, compared with an empty (Milne) universe, $\left(\Omega_{\mathrm{M}}, \Omega_{\Lambda}\right)=(0,0)$. In a matter dominated universe objects are increasingly brighter as a function of redshift in comparison with the "empty" universe. In a $\Lambda$-dominated universe, on the other hand, there is a turning point where the mass density overtakes the effect of the vacuum energy density above $z>1$.

Turning now to extinction due to a homogeneous component of dust, we consider in this work the following scenarios:

- Three combinations of cosmological parameters: $\left(\Omega_{\mathrm{M}}, \Omega_{\Lambda}\right)=(0.3,0.7),(0.2,0)$ and $(1,0)$;

- $R_{V}$ ranging from 5.5 to 9.5 , constant in the interval $0<z<2$;

$-\rho_{\text {dust }}=\rho_{\text {dust }}^{\circ}(1+z)^{\alpha}$, where

$\alpha(z)= \begin{cases}3 \text { for all } z, & \text { Model A } \\ 0 \text { for } z>0.5(3 \text { for lower } z) . & \text { Model B }\end{cases}$

Thus, Model B implies that

$\rho_{\text {dust }}(z>0.5)=\rho_{\text {dust }}(z=0.5)=$ constant,

thus the comoving density increases with cosmic time until $z=0.5$ from which it is constant;

- The extinction scale-length, i.e., the interaction length for photon scattering with dust particles, $\lambda \propto(\sigma$. $\left.\rho_{\text {dust }}^{\circ}\right)^{-1}$ is in the range $(5-300) \cdot\left(\frac{h}{0.65}\right)$ Gpc, where $\sigma$ is the interaction cross-section.

Although models A and B are not necessarily very plausible, they serve as useful limiting cases for more natural 

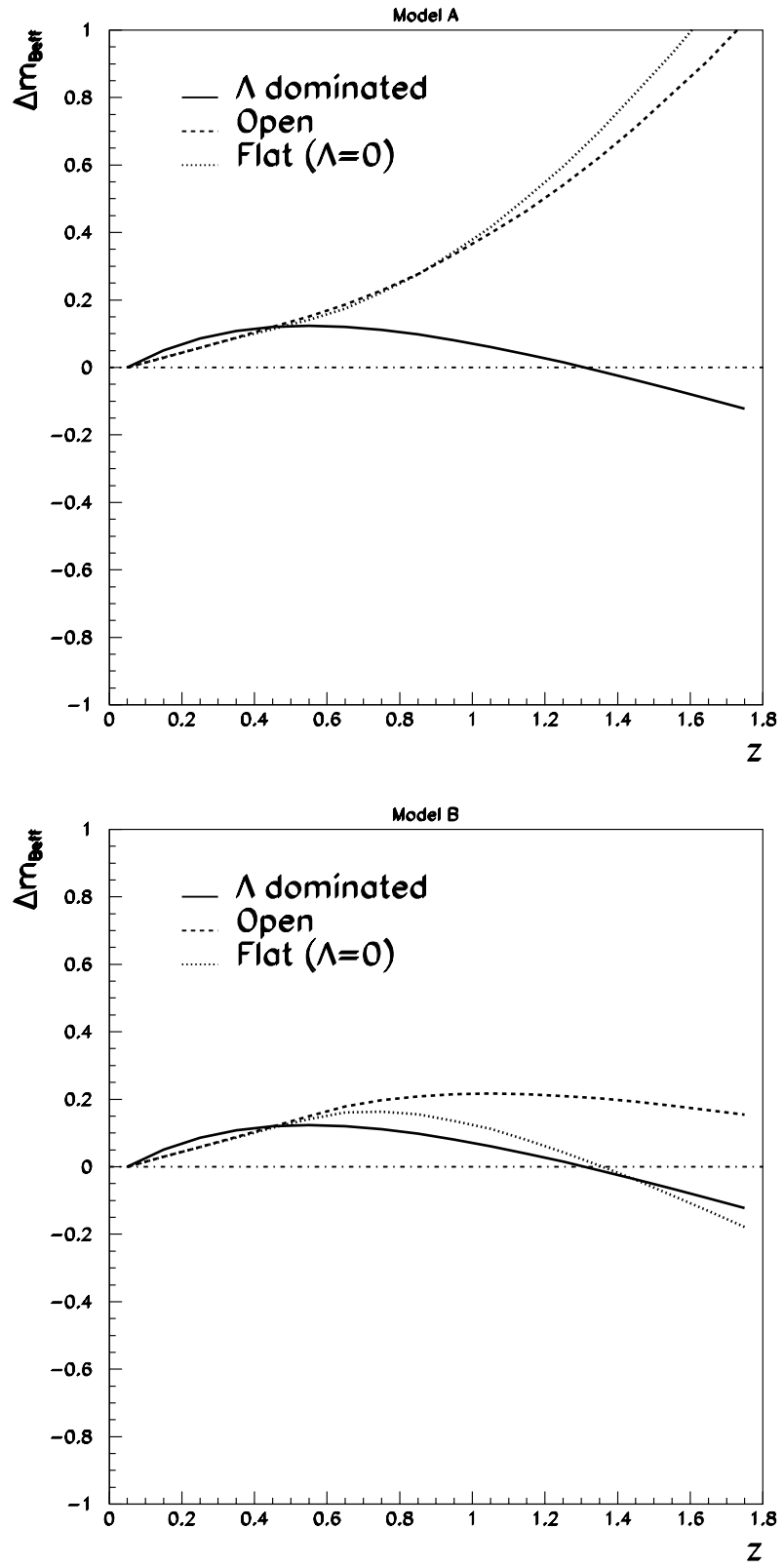

Fig. 3. Differential magnitude for three cosmologies, $\Omega_{\mathrm{M}}, \Omega_{\Lambda}=$ $(0.3,0.7),(0.2,0)$ and $(1,0)$, compared with an empty universe, $\Omega_{\mathrm{M}}, \Omega_{\Lambda}=(0,0)$, where the faintness of the supernovae at $z \sim 0.5$ is achieved by introducing a homogeneous density of intergalactic dust with $R_{V}=5.5$. Upper panel: dust model A. Lower panel: dust model B (see text for details).

scenarios where the dust density is generated and distributed over a finite time scale.

A more detailed calculation would make use of an explicit model for the past star formation rate. However, the predicted evolution of the comoving density between, say, $z \sim 0.8$ and $z \sim 1.7$ corresponds to less than around a factor of 2 (Madau 2000).

In Fig. 3, the apparent faintness of the high-redshift supernovae at $z \sim 0.5$ is forced by introducing a homogeneous grey-dust component. Contrary to the conclusions in (Riess et al. 2001), we find that the restframe

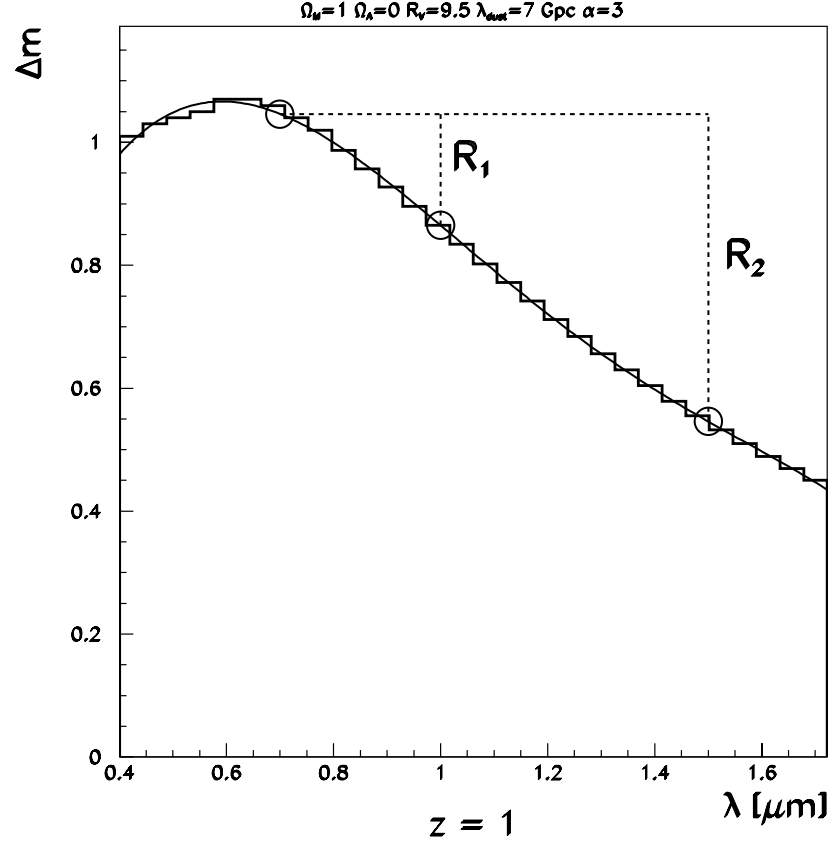

Fig. 4. Monte-Carlo simulation of the differential extinction between $\lambda=0.4$ and $1.7 \mu \mathrm{m}$ for a source at a redshift $z=1$. The solid line shows a fit to a fifth order polynomial. $R_{1}$ is defined to be the magnitude difference in extinction between $0.7 \mu \mathrm{m}$ and $1.0 \mu \mathrm{m}$. $R_{2}$ measures the differential extinction between $0.7 \mu \mathrm{m}$ and $1.5 \mu \mathrm{m}$.

$B$-band brightness of SN1997ff cannot be used to reliably exclude the presence of extragalactic dust at high redshift. The assumption in their analysis is that extinction can be parametrized as $\Delta m_{\text {Beff }}=0.3 \cdot z$ mag in a $\left(\Omega_{\mathrm{M}}, \Omega_{\Lambda}\right)=(0,0)$ universe. This falls in between our models $\mathrm{A}$ and $\mathrm{B}$. If we consider model $\mathrm{B}$ on the other hand, there is less than $0.1 \mathrm{mag}$ difference at $z \sim 1.7$ between the $\Lambda$-dominated and what would be observed in a $\Omega_{\mathrm{M}}=1$ universe with the required dust density to explain the type Ia supernova measurements at $z \sim 0.5$, as shown in lower panel of Fig. 3.

Clearly, accurate spectrophotometric data from a statistical sample of supernovae at this redshift are needed to settle the issue, as described below.

\subsection{Spectrophotometry in the presence of dust}

In order to quantify the induced differential spectral shift due to large dust grains we introduce two quantities: $R_{1}$ and $R_{2} . R_{1}$ is the magnitude difference in extinction between $\lambda=0.7$ and $1.0 \mu \mathrm{m}$. $R_{2}$ measures the differential extinction between $\lambda=0.7$ and $1.5 \mu \mathrm{m}$, as shown in Fig. 4 for a source at $z=1$. Also shown in the figure is a fit of a fifth order polynomial over the wavelength range $\lambda=0.4$ to $1.7 \mu \mathrm{m}$.

The rationale for introducing $R_{1}$ and $R_{2}$ is twofold: 1) the color dependence is almost linear in the range between $\lambda=0.7$ and $1.5 \mu \mathrm{m}$. 2) $R_{1}$ and $R_{2}$ can be measured within the sensitive range of an infrared $(\mathrm{HgCdTe})$ detector. $R_{1}$ can also be measured with a CCD detector. 
A. Goobar et al.: Measuring the properties of extragalactic dust
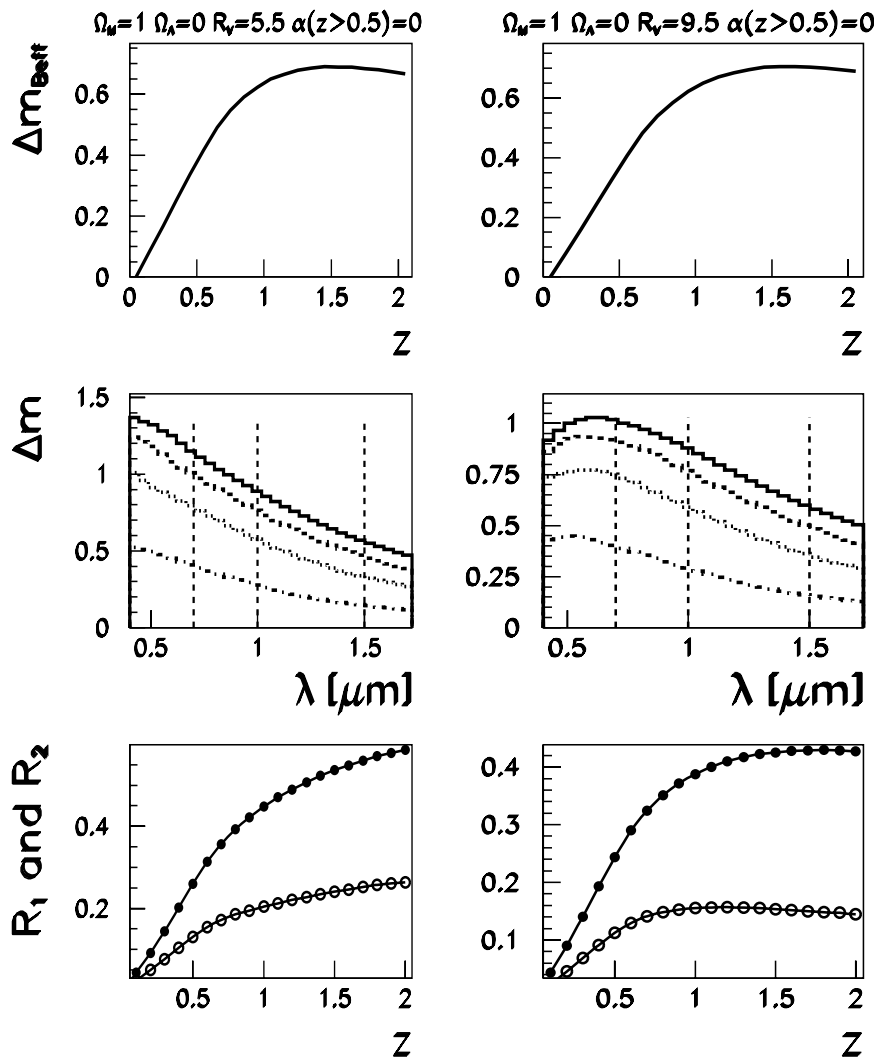

Fig. 5. Dust extinction in model B for $R_{V}=5.5$ (left side) and $R_{V}=9.5$ (right side) in a flat $\left(\Omega_{\mathrm{M}}=1\right)$ universe with the dust density adjusted to generate the required dimming of the restframe $B$-band magnitude from a source at at $z=0.5$. The upper panels show the dimming in the observational band corresponding to the restframe $B$-band. The middle panels show the differential extinction as a function of wavelength for a source at redshifts $z=0.5,1.0,1.5$ and 2.0. The dashed lines show the position of $\lambda=0.7,1.0$ and $1.5 \mu \mathrm{m}$ used to calculate $R_{1}$ and $R_{2}$. The bottom panels show the differential color coefficients $R_{1}$ and $R_{2}$ as a function of redshift.

\section{Grey dust simulations}

\subsection{Open or flat universe with vanishing $\Lambda$}

The idea of Aguirre (1999a, 1999b) was to explain the faintness of high- $z$ type Ia supernovae without invoking a "Dark Energy" component. In such a scenario, an extinction of $0.2-0.5 \mathrm{mag}$ is required to reconcile the supernova data with either an open or flat universe without a cosmological constant. Although perfectly grey dust (i.e., with wavelength-independent absorption) with a fine-tuned redshift distribution possibly can mimick the effects of a cosmological constant, we want to investigate here whether a more natural dust model can do the same. The point to make is that realistic dust firstly has to be related to astrophysical sources, such as star formation, and secondly that it always implies some wavelengthdependence in the absorption and scattering properties.

Figure 5 shows the magnitude and differential extinction as a function of source redshift for model B and
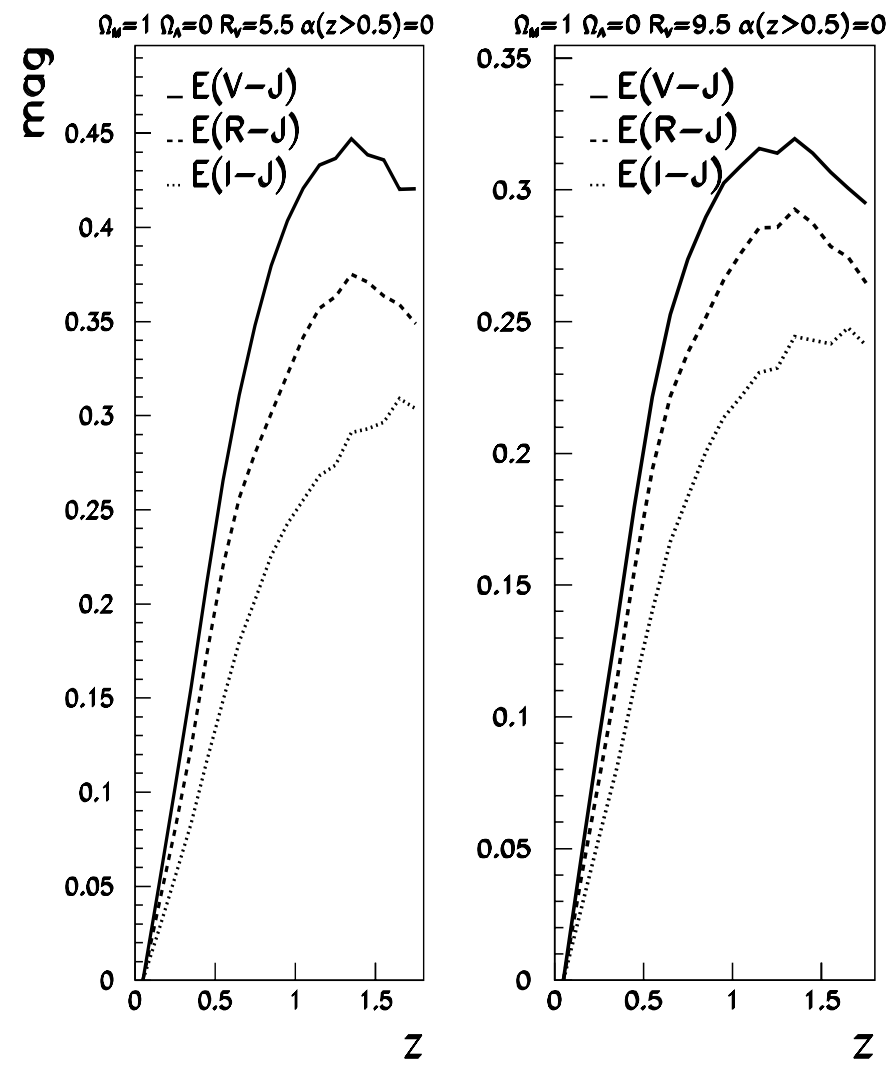

Fig. 6. Color extinctions $E(V-J), E(R-J)$ and $E(I-J)$ for type Ia supernovae in dust model B for $R_{V}=5.5$ (left side) and $R_{V}=9.5$ (right side) in a flat $\left(\Omega_{\mathrm{M}}=1\right)$ universe with the dust density adjusted to generate the required dimming of the restframe $B$-band magnitude from a source at $z=0.5$.

$R_{V}=5.5$ and 9.5 in a flat universe without cosmological constant. The color terms for the highest redshifts are $R_{1} \approx 0.2$ and $R_{2} \approx 0.6$, i.e., clearly measurable with a population of SNe as expected for the proposed SNAP satellite. Figure 6 shows the color extinction (at maximum intensity) in $V-J, R-J$ and $I-J$ for a normal type Ia supernova as a function of redshift ${ }^{2}$. At $z \sim 0.5$, where several supernovae have been observed in the NIR, e.g. (Riess et al. 2000), approximately $0.1-0.15$ and $0.2-0.25$ mag extinction is to be expected in $I-J$ and $V-J$ respectively.

Figure 7 shows the effects of dust extinction in an open universe for $R_{V}=5.5$ and 9.5 where the dust density has been adjusted to fit the observational constraints at $z \sim 0.5$. In both figures, a constant comoving dust density was used in the calculations, i.e. $\alpha=3$ (model A). Figure 8 shows the color extinction for normal type Ia SNe for the same conditions as in Fig. 7.

Figures 9 and 10 show the effect of extinction in an open universe with dust model B. Note that as all the models have been adjusted to match the brightness of type Ia supernovae at $z=0.5$, there is no loss of generality in choosing the redshift at which the dust

${ }^{2}$ All the broadband filters and spectroscopy walvelength scales are in the observer's frame. 

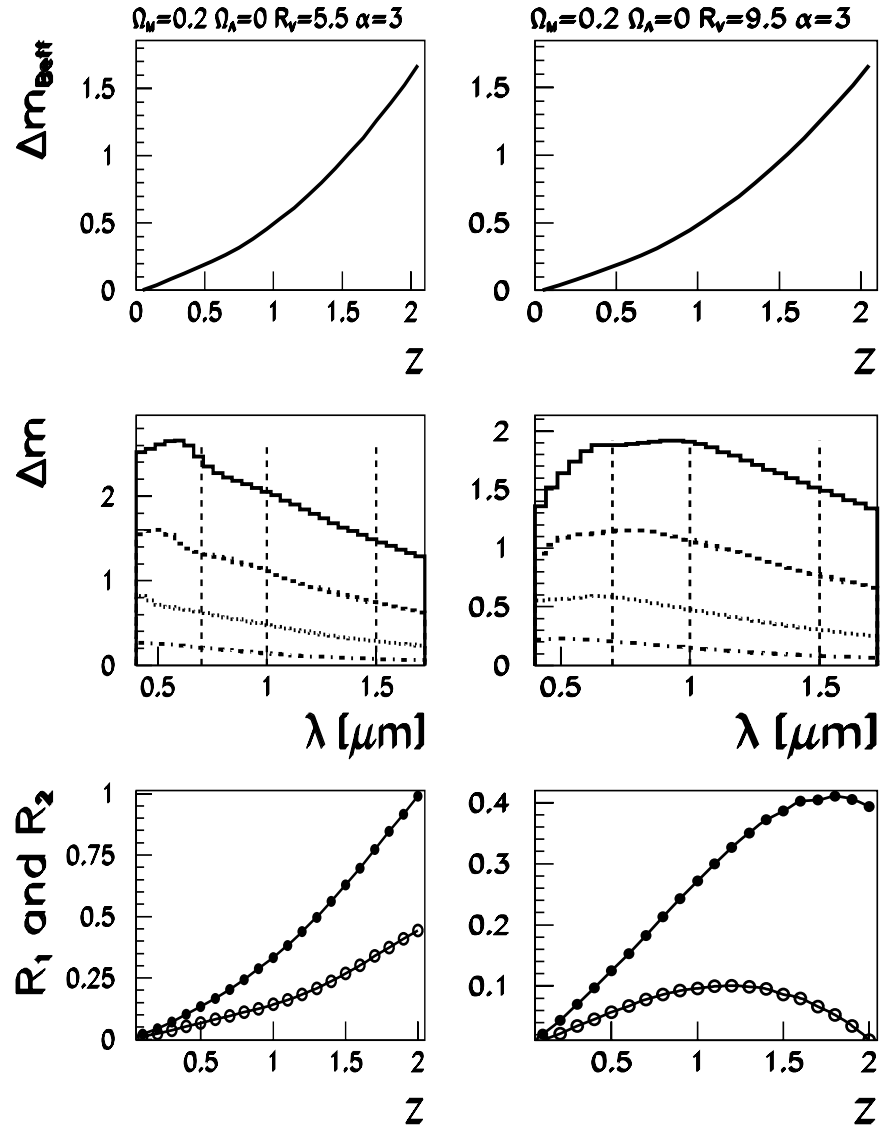

Fig. 7. Dust extinction in model A for $R_{V}=5.5$ (left side) and $R_{V}=9.5$ (right side) in an open $\left(\Omega_{\mathrm{M}}=0.2\right)$ universe with the dust density adjusted to generate the required dimming of the restframe $B$-band magnitude from a source at at $z=0.5$. The upper panels show the dimming in the observational band corresponding to the restframe $B$-band. The middle panels show the differential extinction as a function of wavelength for a source at redshifts $z=0.5,1.0,1.5$ and 2.0. The dashed lines show the position of $\lambda=0.7,1.0$ and $1.5 \mu \mathrm{m}$ used to calculate $R_{1}$ and $R_{2}$. The bottom panels show the differential color coefficients $R_{1}$ and $R_{2}$ as a function of redshift.

density reaches its maximum at $z_{\max }=0.5$ in model $\mathrm{B}$. If $z_{\max }$ is increased, the results will become more similar to model A. If $z_{\max }$ is decreased, the differential extinction decreases somewhat. For example, for $z_{\max }=0.2$ in an open universe, the shapes of the the curves in Figs. 9 and 10 are essentially preserved while the absorption magnitude is approximately half as large as for $z_{\max }=0.5$. If the redshift evolution of the dust density is more rapid, i.e. if there is a burst of production of intergalactic dust, it would be reflected as a sharp discontinuity in the Hubble diagram of type Ia supernovae and their colors.

\subsection{The "standard" $\Lambda$ dominated universe}

Next, we consider the scenario where the extinction due to dust is negligible at $z \sim 0.5$ but that it may introduce a bias in the Hubble diagram in excess of $\delta m=0.02$ for
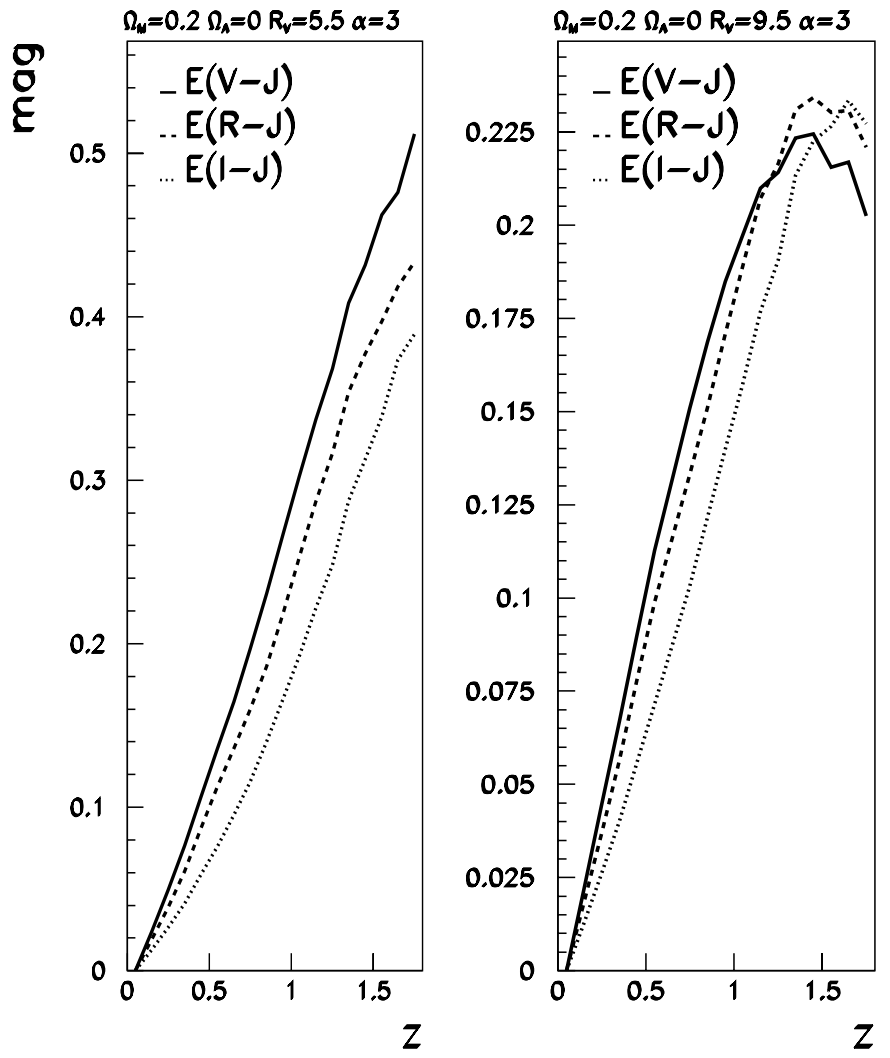

Fig. 8. Color extinctions $E(V-J), E(R-J)$ and $E(I-J)$ for type Ia supernovae in dust model A for $R_{V}=5.5$ (left side) and $R_{V}=9.5$ (right side) in an open $\left(\Omega_{\mathrm{M}}=0.2\right)$ universe with the dust density adjusted to generate the required dimming of the restframe $B$-band magnitude from a source at $z=0.5$.

$z \leq 2$. Thus, we consider a universe where $\Omega_{\mathrm{M}}=0.3$ and $\Omega_{\Lambda}=0.7$, e.g. Figs. 11 and 12 show a case in dust model A. At the limiting redshift range of SNAP a 0.1 magnitude bias in the Hubble diagram results in a approximately a $6 \%$ differential reddening for $R_{2}$ and $2 \%$ in $R_{1}$ for $R_{V}=5.5$. For the same scenario but with $R_{V}=9.5$, $R_{2}$ exceeds 0.01 for $z \gtrsim 0.7$. The corresponding situation in model B can be seen in Figs. 13 and 14 .

The "greyness" of $r>100 \mathrm{~nm}$ dust grains make them completely undetectable with colors bluer than $R$-band in the restframe system. However, for longer wavelengths the extinction leaves a measurable signature in the observed $V-J, R-J$ and $I-J$. This indicates that the $a v$ erage $V-J, R-J$ and $I-J$ colors of normal type Ia supernovae are significantly different with and without the extinction due to large dust grains.

\section{Dust in galaxies along the line of sight}

As dust in galaxies is generally known to cause more reddening than the hypothetical extragalactic "grey" component, intervening galaxies along the line of sight cause less potential danger. Still, it may be of interest to estimate the frequency and size of such encounters. In order to estimate the effects from dust due to intervening galaxies, we 

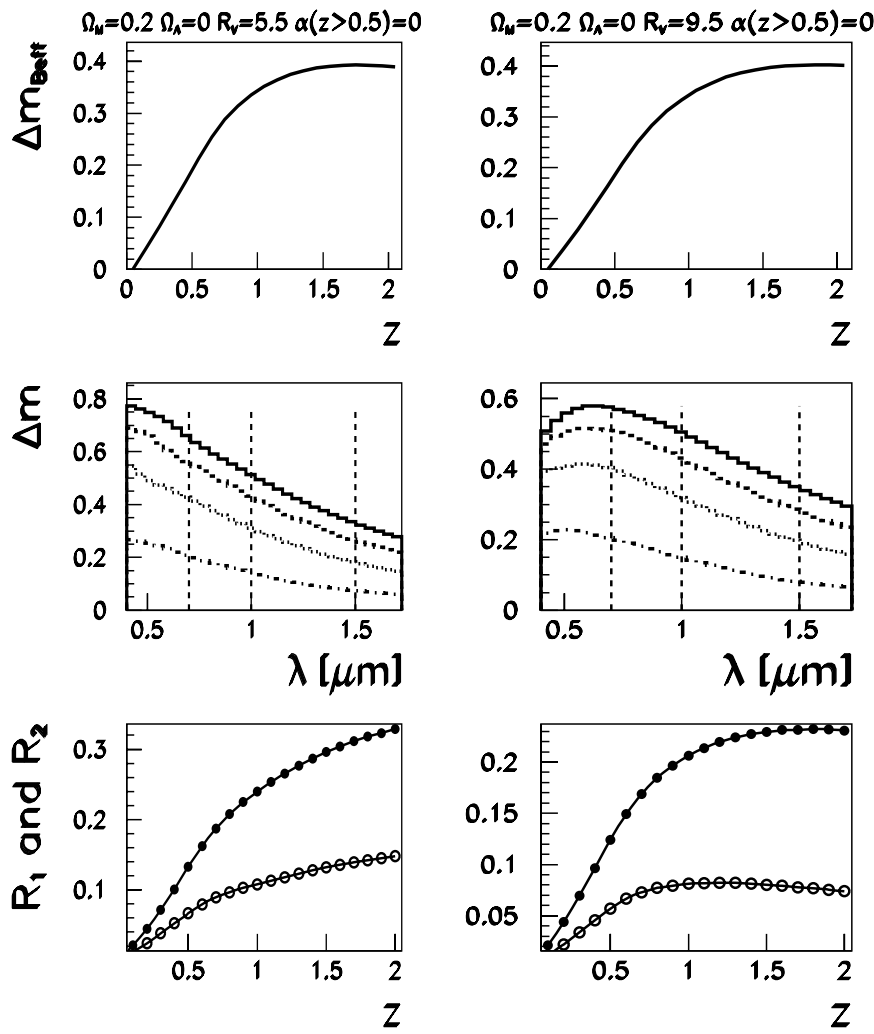

Fig. 9. Dust extinction in model B for $R_{V}=5.5$ (left side) and $R_{V}=9.5$ (right side) in an open $\left(\Omega_{\mathrm{M}}=0.2\right)$ universe with the dust density adjusted to generate the required dimming of the restframe $B$-band magnitude from a source at at $z=0.5$. The upper panels show the dimming in the observational band corresponding to the restframe $B$-band. The middle panels show the differential extinction as a function of wavelength for a source at redshifts $z=0.5,1.0,1.5$ and 2.0. The dashed lines show the position of $\lambda=0.7,1.0$ and $1.5 \mu \mathrm{m}$ used to calculate $R_{1}$ and $R_{2}$. The bottom panels show the differential color coefficients $R_{1}$ and $R_{2}$ as a function of redshift.

have thus performed a number of ray-tracing Monte-Carlo simulations. We follow a light-ray between the source and the observer by sending the ray through a series of spherical cells. Each cell encompasses a galaxy at the center and the size of the cell is computed to accurately represent the number density of galaxies, see (Goobar et al. 2002) for a more detailed description of the Monte-Carlo method.

In Fig. 15 we have plotted the smallest galaxy impact parameters for 10000 light-rays originating at redshifts $z=0.5, z=1$ and $z=1.5$ in a $\Lambda$-dominated universe, $\left(\Omega_{\mathrm{M}}, \Omega_{\Lambda}\right)=(0.3,0.7)$. We see that the probability for a light-ray to pass closer than $10 \mathrm{kpc}$ to a galaxy center is $0.6 \%, 1.5 \%$ and $3.0 \%$ for source redshifts of $z=0.5, z=1$ and $z=1.5$ respectively.

In order to estimate the magnitude of the effect, we model the dust distribution in spiral galaxies by a double exponential disk with random inclination,

$\rho_{\text {dust }}=\rho_{\text {dust }}^{0} \mathrm{e}^{-r / r_{0}} \mathrm{e}^{-\zeta / \zeta_{0}}$.
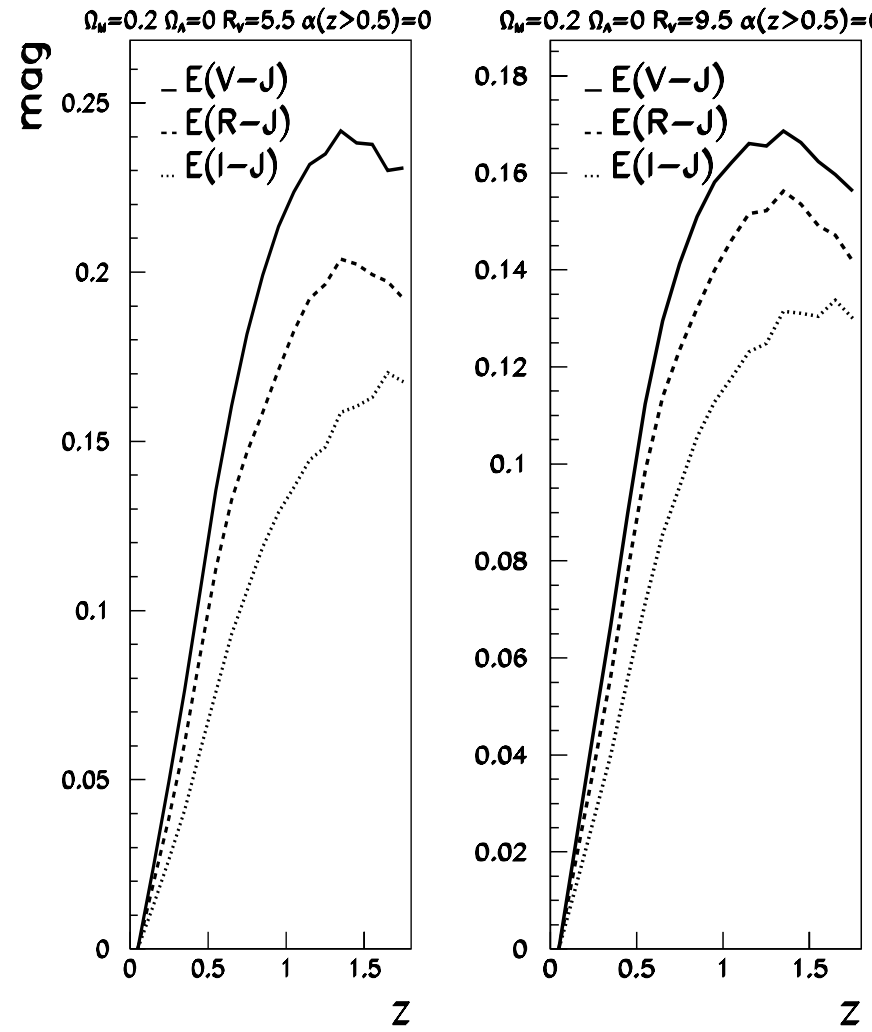

Fig. 10. Color extinctions $E(V-J), E(R-J)$ and $E(I-J)$ for type Ia supernovae in dust model B for $R_{V}=5.5$ (left side) and $R_{V}=9.5$ (right side) in an open $\left(\Omega_{\mathrm{M}}=0.2\right)$ universe with the dust density adjusted to generate the required dimming of the restframe $B$-band magnitude from a source at $z=0.5$.

For simplicity, we assume that the dust-density scalelength in the $\zeta$-direction, $\zeta_{0}$, is constant $(0.1 \mathrm{kpc})$ whereas the radial scale-length, $r_{0}$, is proportional to the squareroot of the luminosity of the galaxy, i.e., we assume that the distribution of dust follows the distribution of stars in the galaxy. For an $L_{*}$ galaxy, we use a scale-length $r_{0}=5 \mathrm{kpc}$. The dust-disk is truncated at $\zeta=3 \mathrm{kpc}$ and $r=20 \mathrm{kpc}$. We assume that the fraction of galaxies that are spirals is given by

$f_{\mathrm{s}}(z)=f_{\mathrm{s}}(0)+q \cdot z$

where $f_{\mathrm{s}}(0)=0.7$ and $q=0.05$. For an extinction scalelength at the galaxy centers of $1 \mathrm{kpc}$, we obtain a probability for a supernova at $z \sim 1$ to be demagnified by more than 0.02 mag by dust in intervening galaxies of $\sim 0.33 \%$. However, this number is very sensitive to the normalization of the dust density since the absorption expressed in magnitudes is inversely proportional to the extinction scale-length of the absorption. Since a light-ray passing close to a galaxy will be magnified due to gravitational lensing effects, we expect some correlation of the demagnification due to dust and the magnification due to gravitational lensing. In Fig. 16, the magnification from lensing is plotted against the demagnification from dust in units of the inverse extinction scale-length. To obtain the 

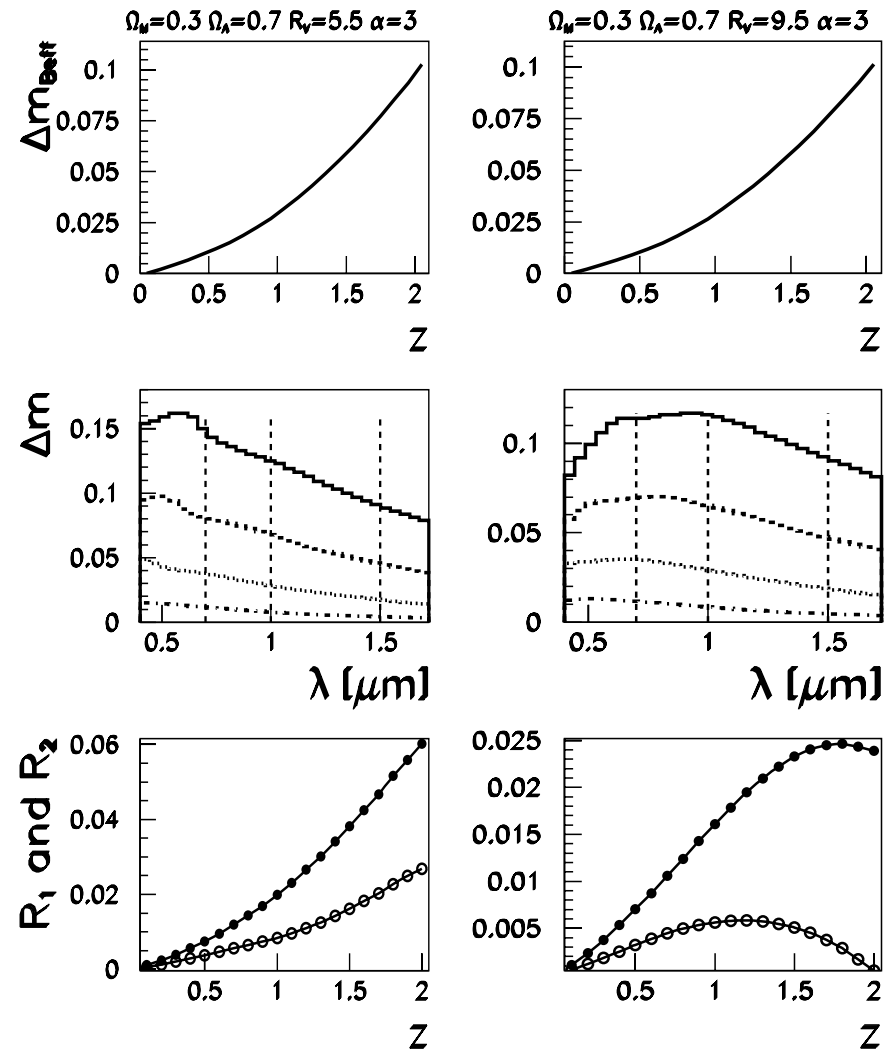

Fig. 11. Dust extinction in model $\mathrm{A}$ for $R_{V}=5.5$ (left side) and $R_{V}=9.5$ (right side) in a flat $\Lambda$-dominated universe with the dust density adjusted to generate about $0.1 \mathrm{mag}$ dimming in the observed band corresponding to the restframe $B$-band magnitude from a source the the limiting SNAP depth $\left(\lambda_{\text {dust }}=300 \cdot\left(\frac{0.65}{h}\right) \mathrm{Gpc}\right)$. The upper panels show the dimming in the observational band corresponding to the restframe $B$-band. The middle panels show the differential extinction as a function of wavelength for a source at redshifts $z=0.5,1.0,1.5$ and 2.0. The dashed lines show the position of $\lambda=0.7,1.0$ and $1.5 \mu \mathrm{m}$ used to calculate $R_{1}$ and $R_{2}$. The bottom panels show the differential color coefficients $R_{1}$ and $R_{2}$ as a function of redshift.

demagnification for a specific scale-length, one should divide the numbers on the $x$-axis in Fig. 16 with the extinction scale-length expressed in kpc.

\section{Extinction in host galaxies}

Type Ia supernovae occur in both late and early type galaxies. In the case of late type hosts, we need to take obscuration due to dust in the host galaxy into account. We model the distribution of dust in late type host according to Eq. (3), and the generation of type Ia SNe as two different components, following (Hatano et al. 1998). In the disk component, the generation of type Ia SNe follows the dust distribution only that the scale-length in the $\zeta$-direction is increased to $0.35 \mathrm{kpc}$. There is also a bulge component where the SNe are spherically distributed as $\left(R^{3}+0.7^{3}\right)^{-1}$, where the distance from galactic center, $R$, is expressed in kpc and the bulge is truncated at $3 \mathrm{kpc}$.
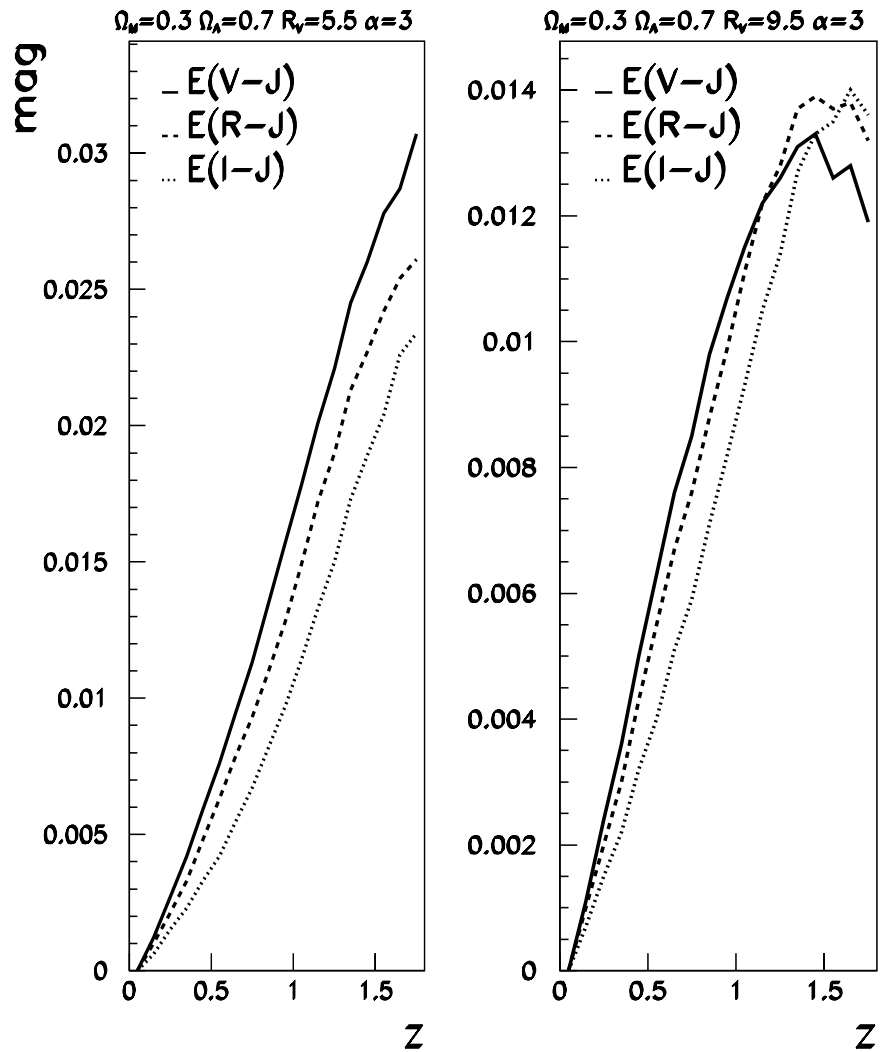

Fig. 12. Color extinctions $E(V-J), E(R-J)$ and $E(I-J)$ for type Ia supernovae in dust model A for $R_{V}=5.5$ (left side) and $R_{V}=9.5$ (right side) in a flat $\Lambda$-dominated universe with the dust density adjusted to generate about 0.1 mag dimming in the observed band corresponding to the restframe $B$-band magnitude from a source the limiting SNAP depth.

We assume that the probability for a SNe to occur in the bulge is $1 / 8$ of the probability in the disk. Following (Matteucci \& Recchi 2001), we estimate the probability for a type Ia SNe to occur in an early type galaxy to be

$p_{\mathrm{E}}(z)=p_{\mathrm{E}}(0)+q \cdot z$

where $p_{\mathrm{E}}(0)=0.5$ and $q=0.125$. In Fig. 17, we plot the obscuration due to host galaxy dust for 10000 type Ia SNe. Note that the magnitude of the results is very sensitive to the normalization of the dust density. To obtain the demagnification, divide the numbers on the $x$-axis with the extinction scale-length expressed in kpc. For an extinction scale-length of $1 \mathrm{kpc}, \sim 2600$ out of 10000 sources are demagnified by more than 0.02 mag by host galactic dust.

\section{Discussion}

The effects of grey dust extinction capable of biasing the results of experiments such as the proposed SNAP satellite can be diagnosed through accurate relative spectrophotometry or broadband photometry at the $1 \%$ level in the $0.7-1.5 \mu \mathrm{m}$ wavelength range, as shown in e.g. Figs. 13 and 14. This would allow tests for intergalactic dust obscuration affecting the measurement of high- $z$ supernovae 

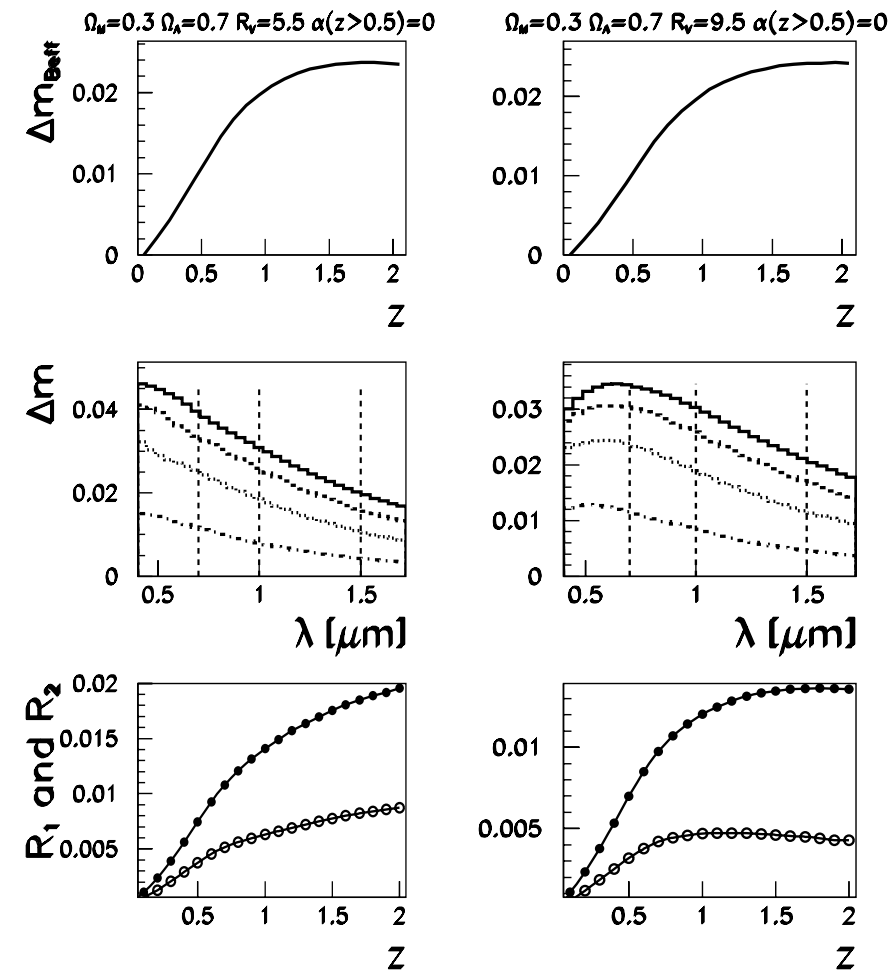

Fig. 13. Dust extinction in model B for $R_{V}=5.5$ (left side) and $R_{V}=9.5$ (right side) in a flat $\Lambda$-dominated universe with the dust density adjusted to generate about 0.02 mag dimming in the observed band corresponding to the restframe $B$-band magnitude from a source the the limiting SNAP depth $\left(\lambda_{\text {dust }}=300 \cdot\left(\frac{0.65}{h}\right) \mathrm{Gpc}\right)$. The upper panels show the dimming in the observational band corresponding to the restframe $B$-band. The middle panels show the differential extinction as a function of wavelength for a source at redshifts $z=0.5,1.0,1.5$ and 2.0. The dashed lines show the position of $\lambda=0.7,1.0$ and $1.5 \mu \mathrm{m}$ used to calculate $R_{1}$ and $R_{2}$. The bottom panels show the differential color coefficients $R_{1}$ and $R_{2}$ as a function of redshift.

up to $\Delta m \sim 0.02$, the target for systematic uncertainties for SNAP.

Achieving 1-2\% relative spectrophotometric accuracy in the optical and NIR for $22-29$ mag objects is rather challenging. As the measurements will rely on a large number of homogeneous high-redshift sources, it is not required to get $S / N=50-100$ for individual objects. On the other hand, the instrument calibration must be within $1 \%$ over the course of at least one year for the case of SNAP. This can be accomplished e.g. through repeated observations of hot galactic white dwarfs (Bohlin 1996; Finley et al. 1997). In the Rayleigh-Jeans limit, $T_{\text {eff }} \gg 20000 \mathrm{~K}$, the color of the calibrators become independent of temperature and are therefore ideal for relative calibration.

Significant progress in examining the possible bias due to grey dust in the published type Ia supernova Hubble diagram can already be made with existing NIR instruments. If the faintness of type Ia $\mathrm{SNe}$ at high- $z$ is to be attributed to grey dust obscuration as
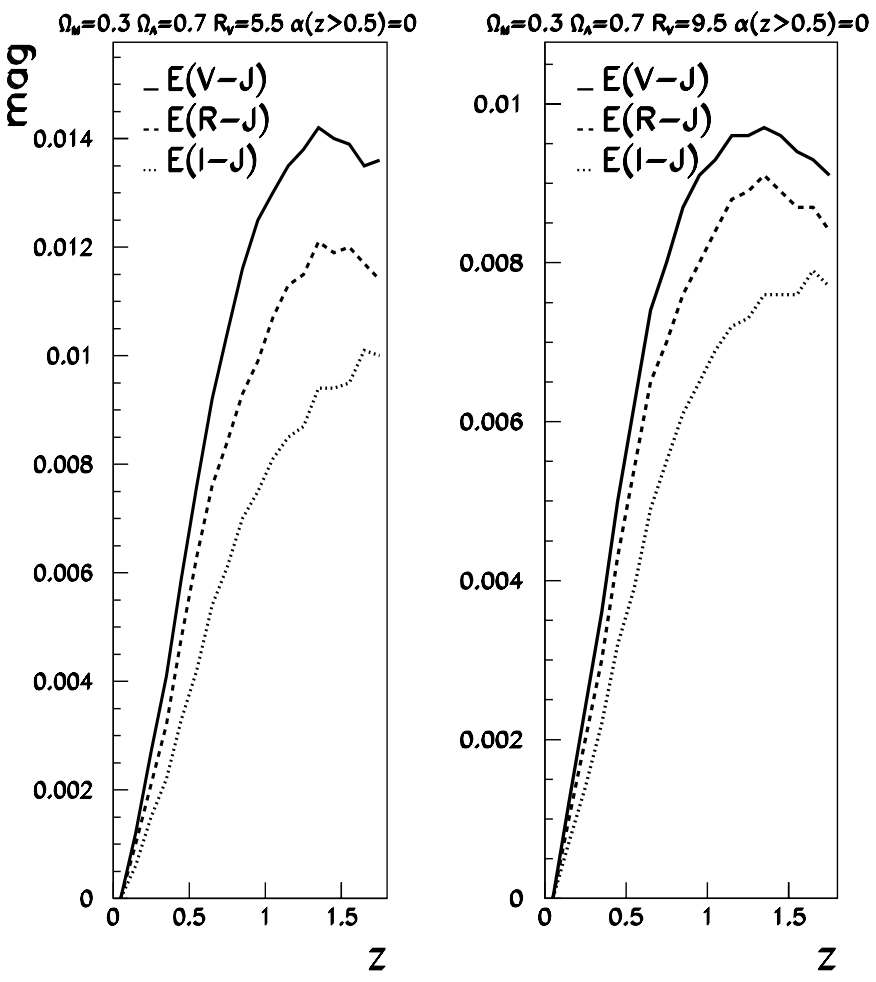

Fig. 14. Color extinctions $E(V-J), E(R-J)$ and $E(I-J)$ for type Ia supernovae in dust model B for $R_{V}=5.5$ (left side) and $R_{V}=9.5$ (right side) in a flat $\Lambda$-dominated universe with the dust density adjusted to generate about 0.02 mag dimming in the observed band corresponding to the restframe $B$-band magnitude from a source the limiting SNAP depth.

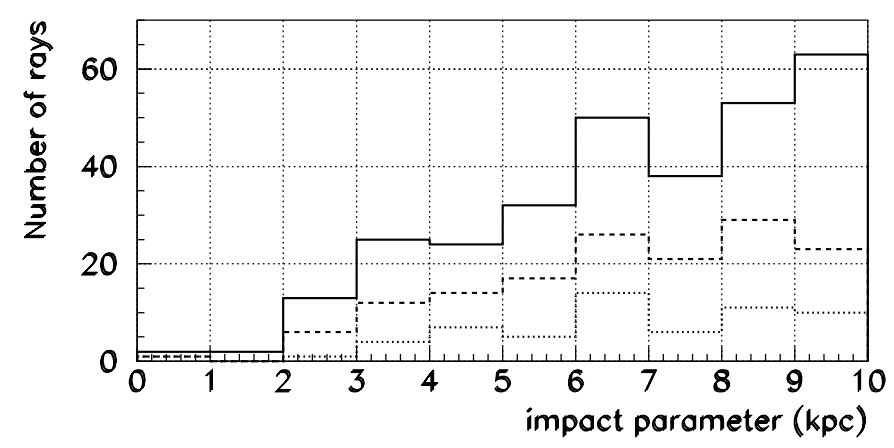

Fig. 15. The distribution of smallest galaxy impact parameters for 10000 sources at redshifts $z=0.5$ (dotted line), $z=1$ (dashed line) and $z=1.5$ (full line) in a $\Lambda$-dominated universe, $\left(\Omega_{\mathrm{M}}, \Omega_{\Lambda}\right)=(0.3,0.7)$.

opposed to the cosmological explanation a color extinction $E(R-J)^{\text {obs }} \gtrsim 0.1 \mathrm{mag}$ is to be expected for sources at $z \sim 0.5$. Testing this possibility is within reach with ground based facilities.

We have also examined the possibility of extinction as the beam of high redshift passes through foreground galaxies. This was found to be a relatively small effect, only causing $>0.02$ mag dimming for less than $1 \%$ of the 


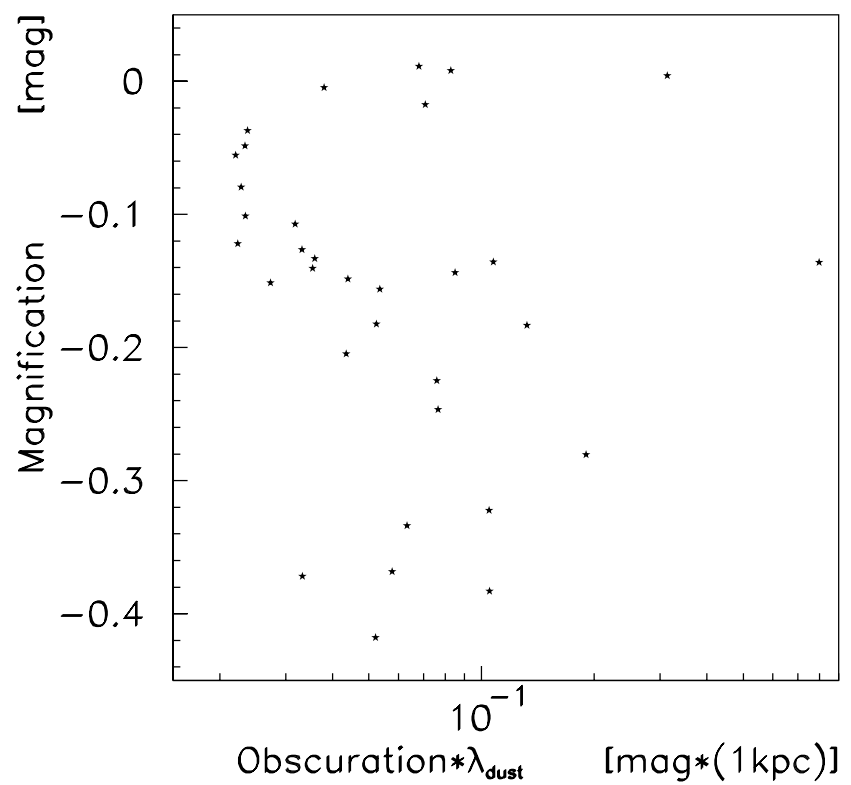

Fig. 16. The correlation of the demagnification due to dust and the magnification due to gravitational lensing for the 33 out of 10000 sources at $z=1$ which are demagnified more than $0.02 \mathrm{mag}$ by intervening galactic dust for an extinction scale-length at the galaxy centers of $1 \mathrm{kpc}$. To obtain the demagnification for an arbitrary scale-length, divide the numbers on the $x$-axis with the scale-length expressed in kpc.

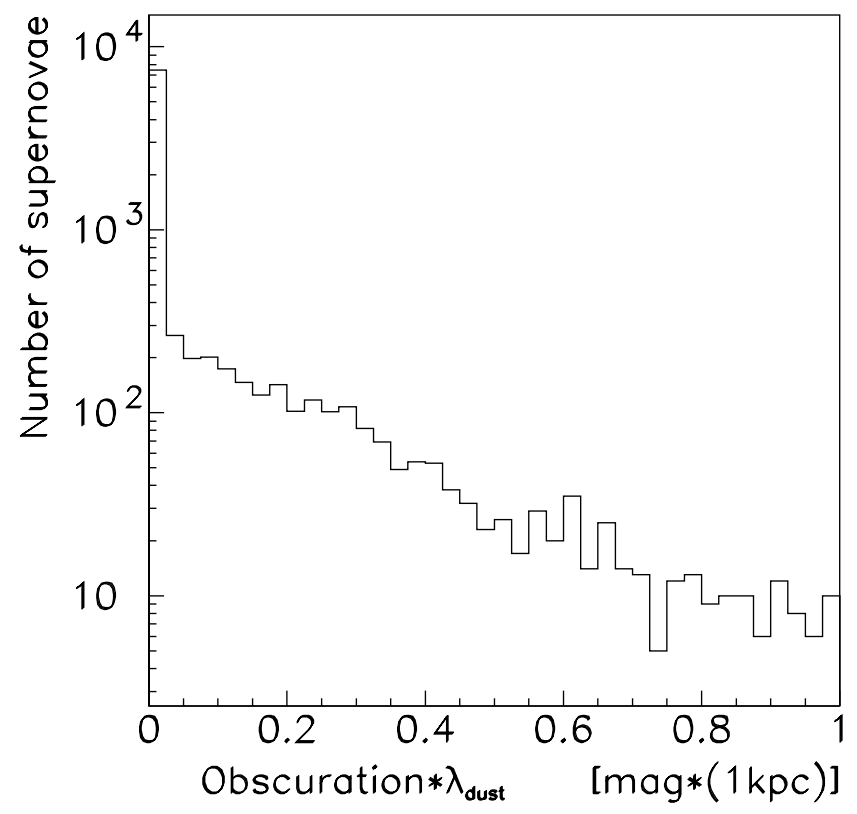

Fig. 17. The demagnification due to dust in the host galaxy. For an extinction scale-length of $1 \mathrm{kpc}, \sim 2600$ out of 10000 sources are demagnified by more than $0.02 \mathrm{mag}$ by host galactic dust.

sources at $z=1$, whereas extinction in the host galaxy potentially is a more serious problem.

However, in general we expect dust in galaxies along the line of sight and in host galaxies to have $R_{V} \sim 3$, causing considerably more reddening than an intergalactic "grey" dust component. Thus, with high accuracy spectrophotometry, it should be possible to control the effects from extinction in galaxies.

Acknowledgements. The authors would like to thank Anthony Aguirre, Alex Kim, and Peter Nugent for useful discussions and Serena Nobili for a careful reading of the manuscript. AG is a Royal Swedish Academy Research Fellow supported by a grant from the Knut and Alice Wallenberg Foundation. LB would like to thank the Swedish Research Council for financial support.

\section{References}

Aguirre, A. 1999, ApJ, 512, L19

Aguirre, A. 1999, ApJ, 525, 583

Aguirre, A., \& Haiman, Z. 2000, ApJ, 532, 28

Bahcall, N. A., \& Fan, X. 1998, ApJ, 504, 1

Balbi, A., Ade, P., Bock, J., et al. 2000, ApJ, 545, 1; erratum: 2001, ApJ, 558, 145

Bohlin, R. C. 1996, AJ, 111, 1743

Cardelli, J. A., Clayton, G. C., \& Mathis, J. S. 1989, ApJ, 345, 245

de Bernardis, P., Ade, P. A. R., Bock, J. J., et al. Nature, 2000, 404, 955

Draine, B., \& Lee, H. 1984, ApJ, 285, 89

Finley, D. S., Koester, D., \& Basri, G. 1997, ApJ, 488, 375

Goliath, M., Amanullah, R., Astier, P., Goobar, A., \& Pain, R. 2001, A\&A, in press [astro-ph/0104009]

Goobar, A., et al. 2002, the SNOC Monte-Carlo package, in preparation

Hatano, K., Branch, D., \& Deaton, J. 1998, ApJ, 502, 177

Ivanov, V. D., Borissova, J., \& Vanzi, L. 2000, ApJ, 542, 588

Madau, P. 2000, Proc. of the Nobel Symposium, Particle Physics and the Universe, ed. L. Bergström, P. Carlson, \& C. Fransson, Phys. Scr., T85, 156

Matteucci, F., \& Recchi, S. 2001, ApJ, 558, 351

Mörtsell, E., Goobar, A., \& Bergström, L. 2001, ApJ, 559, 53

Nugent, P., et al. 2002, submitted to PASP

Peacock, J. A., Cole, S., Norberg, P., et al. 2001, Nature, 410, 169

Perlmutter, S., Aldering, G., Goldhaber, G., et al. 1999, ApJ, 517,565

Perlmutter, S., et al. 2000, the SuperNova Acceleration Probe, http://snap.lbl.gov

Pryke, C., et al. 2001, submitted to ApJ, preprint [astro-ph/0104490]

Riess, A. G., Filippenko, A. V., Challis, P., et al. 1998, AJ, 116, 1009

Riess, A., Filippenko, A. V., Liu, M. C., et al. 2000, ApJ, 536, 62

Riess, A., Nugent, P. E., Gilliland, R. L., et al. 2001, ApJ, 560, 49

Steinhardt, P. J. 2000, Proc. of the Nobel Symposium, Particle Physics and the Universe, ed. L. Bergström, P. Carlson, \& C. Fransson, Phys. Scr., T85, 177

Vanden Berk, D. E., Richards, G. T., Bauer, A., et al. 2001, AJ, 122, 549 